\title{
Precise Formation-Flying Telescope in Target-Centric Orbit: the Solar Case
}

\author{
Pascal Saint-Hilaire ${ }^{1}$ (D) . Jeffrey E. Marchese ${ }^{1}$
}

Published online: 22 September 2020

(C) The Author(s) 2020

\begin{abstract}
We present the general concept of a telescope with optics and detectors mounted on two separate spacecrafts, in orbit around the telescope's target (scopocentric or target-centric orbit), and using propulsion to maintain the Target-Optics-Detector alignment and Optics-Detector distance. Specifically, we study the case of such a telescope with the Sun as the target, orbiting at $\sim 1$ AU. We present a simple differential acceleration budget for maintaining Target-Optics-Detector alignment and Optics-Detector distance, backed by simulations of the orbital dynamics, including solar radiation pressure and influence of the planets. Of prime interest are heliocentric orbits (such as Earth-trailing/leading orbits or Distant Retrograde Orbits), where thrust requirement to maintain formation is primarily in a single direction (either sunward or anti-sunward), can be quite minuscule (a few $\mathrm{m} / \mathrm{s} / \mathrm{year}$ ), and preferably met by constant-thrust engines such as solar electric propulsion or even by solar sailing via simple extendable and/or orientable flaps or rudders.
\end{abstract}

Keywords Formation flying · Precise Formation Flying · Heliocentric · Telescope

\section{Introduction}

To date, spaceborne telescope boom lengths have rarely exceeded $15 \mathrm{~m}$, and achieving much greater separation distances in space between optics and detectors will likely require precise formation flying (PFF). The European Space Agency's Proba$3[1,2]$, currently scheduled to be launched in 2022, attempts such a feat, with a coronograph spacecraft separated from a telescope spacecraft by a distance of $\approx 150$ $\mathrm{m}$ in high Earth orbit. The Sun-coronograph-telescope alignment is planned to last about six hours during each $\sim$ day-long length of the highly eccentric orbit. Besides

Pascal Saint-Hilaire

pascal@ssl.berkeley.edu

1 Space Sciences Laboratory, University of California, Berkeley, 7, Gauss Way,

Berkeley, 94720, CA, USA 
an occulter-telescope pair, there are several other possible applications for such a concept. For example in the X-ray regime, the optics can be a Fresnel Zone Plate [3], a focusing optics mirror (the longer focal length enables shallower incidence angle on the mirrors, allowing higher energies to be reached), or a coded-mask or (rotating) grid allowing e.g. higher angular resolutions.

We are interested in the case of the Sun as a target, with the Optics spacecraft and Detector spacecraft in a "scopocentric" orbit around it (Fig.1), and we will use the term "Precise Formation Flying," or PFF, to refer to the combination of TargetOptics-Detector alignment maintenance and Optics-Detector distance maintenance. This paper strives to make the case for scientists requiring large optics-to-detectors distances for the next generation of space instrumention that the time is nigh, and that a solar application is probably an easy first step.

Section "Differential Accelerations" details the various forces acting on each spacecraft, Section "Simulations" describes the simulations done to confirm the theory, Section "Discussion" discusses the results and their possible applications with current or soon-to-be available launch capabilities for CubeSats and SmallSats. Finally, Section "Conclusions" summarizes and concludes this work.

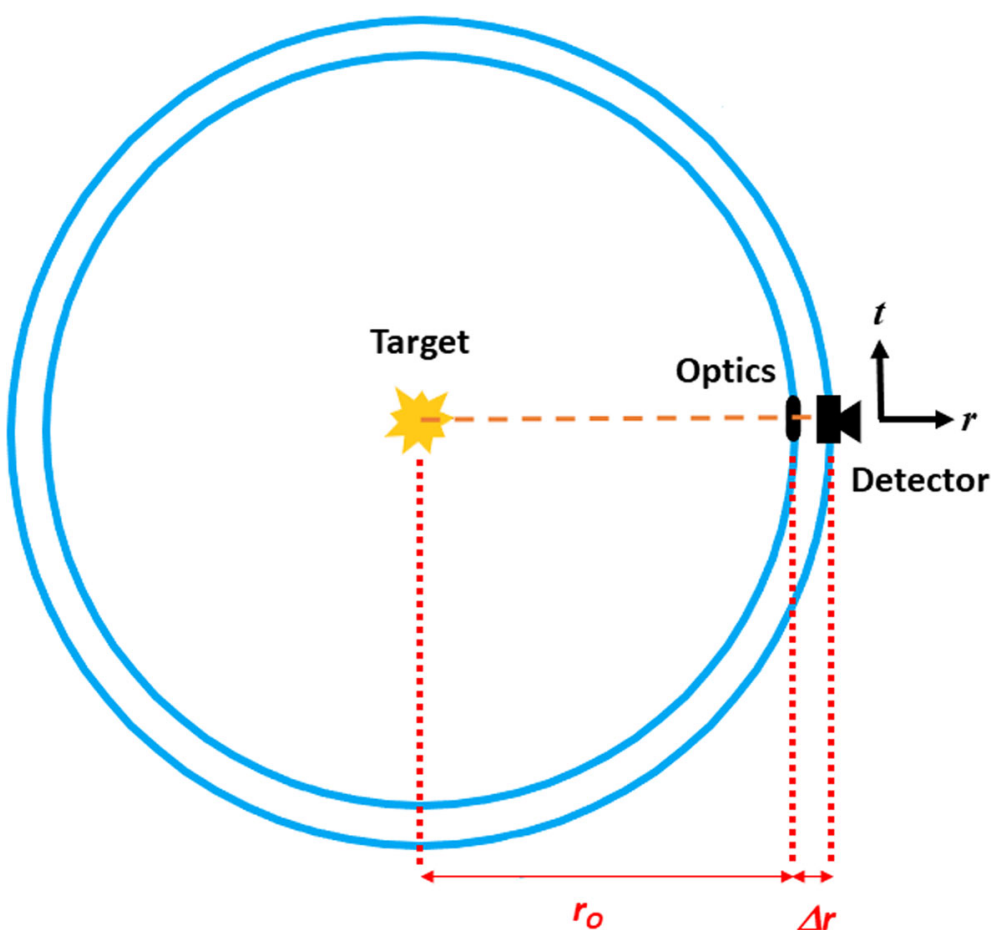

Fig. 1 Diagram and notations (here, a circular orbit). Throughout the orbit, target, Optics spacecraft and Detector spacecraft must remain aligned at all times, and the Optics-Detector separation must remain constant 


\section{Differential Accelerations}

To maintain formation, either the Optics spacecraft or the Detector spacecraft must fight against the (often minute, but with long-term consequences) differences in the total accelerations they experience (solar radiation pressure, orbital/tidal effects from the orbited body/target, and tidal effects from nearby celestial bodies).

We will refer to the spacecraft closest to the the target as "Optics spacecraft", and the spacecraft furthest away as "Detector spacecraft". In many cases, the Optics spacecraft can be thought of as the spacecraft carrying the optics of the telescope, and the Detector spacecraft as the one carrying the detectors. Obviously, there can be variations: the Optics spacecraft can carry an occulter while the Detector spacecraft carries both optics and detectors (e.g. Proba-3). We present here simple analytical, acceleration budgets, mostly in the form of differential accelerations $\delta \vec{a}_{D}$ that the Detector spacecraft must exert to maintain the formation. It has radial component $\delta a_{D, r}$ along the target-optics-detector axis, and tangential component $\delta a_{D, t}$ perpendicular to it, in the orbital plane. A negative $\delta a_{D, r}$ hence implies a sunward thrust requirement by the Detector spacecraft (or anti-sunward by the Optics spacecraft).

Most of the following is applicable when the target is any celestial body that can be orbited (e.g. a planet), but we will concentrate on the case of the Sun as a target. We will assume that the Optics spacecraft is in keplerian orbit, and that the Detector spacecraft does all the necessary thrusting to maintain the Target-Optics-Detector alignment and Optics-Detector distance throughout the orbit. The calculations and conclusions are of course almost completely interchangeable between the two spacecrafts, though considerations such as having a thruster in the optics path might constrain specific implementations.

Section "Thrust the Detector Spacecraft must Exert to Sustain its non-Keplerian ("NK") Orbit" deals with compensating for the non-keplerian orbit of the Detector spacecraft, Section "Differential Acceleration due to Tidal Effects from Other Celestial Bodies" deals with compensating for the disruptive effects from other bodies than the one being orbited, and Section "Differential Acceleration due to Solar Radiation Pressure" deals with the effects of radiation pressure (which dominate when the formation-flying telescope is sufficiently far away from all celestial bodies). Finally, Section "Differential Acceleration due to Drag in Ram Direction" deals with interplanetary and atmospheric drags.

\section{Thrust the Detector Spacecraft must Exert to Sustain its non-Keplerian ("NK") Orbit}

Linearized relative equation of motions in circular orbits have been presented by Clohessy-Wiltshire [4] for the case of circular orbits. We will jump directly to the elliptical case, using the linearized equations from [5] (or see Section 5.6 of [6]):

$$
\delta a_{D, r}=\ddot{x}-2 \dot{\theta}_{O} \dot{y}-\ddot{\theta}_{O} y-\left(\dot{\theta}_{O}^{2}+2 \frac{G M}{r_{O}^{3}}\right) x
$$




$$
\begin{aligned}
& \delta a_{D, t}=\ddot{y}+2 \dot{\theta}_{O} \dot{x}+\ddot{\theta}_{O} x-\left(\dot{\theta}_{O}^{2}+2 \frac{G M}{r_{O}^{3}}\right) y \\
& \delta a_{D, n}=\ddot{z}+\frac{G M}{r_{O}^{3}} z
\end{aligned}
$$

Where $x, y, z$ are the radial, tangential, and normal components of the Detector spacecraft relative position with respect to the Optics spacecraft, and $a_{D}$ the thrust (acceleration) delivered by the Detector spacecraft. The Optics spacecraft is in Keplerian orbit, hence $r_{O}^{2} \dot{\theta}_{O}=h$, with $h=\sqrt{G M a_{O}\left(1-e_{O}^{2}\right)}$ the (constant) angular momentum, $a_{O}$ the semi-major axis, and $e_{O}$ the eccentricity. To maintain the TargetOptics-Detector alignment and Optics-Detector distance, $\ddot{x} \equiv \ddot{y} \equiv \ddot{z} \equiv \dot{x} \equiv \dot{y} \equiv$ $\dot{z} \equiv y \equiv z \equiv 0$, and $x$ is a constant kept at $\Delta r$, leading to the thrust requirement on the Detector spacecraft:

$$
\begin{aligned}
\delta a_{D, r}^{\mathrm{NK}} & =-\frac{G M}{r_{O}^{3}}\left(2+\frac{r_{O}\left(1+e_{O} \cos \theta_{O}\right)^{2}}{a_{O}\left(1-e_{O}^{2}\right)}\right) \Delta r \\
& =-\frac{G M}{r_{O}^{3}} \Delta r\left(\frac{1+e_{O} \cos \theta_{O}}{1-e_{O}^{2}}\right)^{3}\left(3+e_{O} \cos \theta_{O}\right) \\
\delta a_{D, t}^{\mathrm{NK}} & =-2 \frac{G M}{r_{O}^{3}} \Delta r e_{O} \sin \theta_{O}
\end{aligned}
$$

If the Detector spacecraft applies the above thrusts, then it will orbit the target with the same period as the Optics spacecraft, but $\Delta r$ further away, and preserving the Target-Optics-Detector alignment. The Detector spacecraft orbit is faster than if it were freely-coasting. In the circular $\left(e_{O}=0\right)$ case, $\delta a_{D, r}^{\mathrm{NK}}=-1.2 \times 10^{-11} \mathrm{~m} / \mathrm{s}^{2}=$ $-0.38 \mathrm{~mm} / \mathrm{s} / \mathrm{yr}$ for $a_{O}=1 \mathrm{AU}, M=M_{\text {sun }}$, and $\Delta r=100 \mathrm{~m}$.

The above equations are valid for $\Delta r \ll r_{O}$. As some applications requiring extremely large spacecraft separations are not inconceivable, though certainly technologically extremely difficult (e.g. a Fresnel lens for $\gamma$-rays observations), we also present exact solutions in Appendix A that are valid for any $\Delta r<r_{O}$.

Figure 2 displays the orbital variation of the required accelerations for the case $a_{O}=1 \mathrm{AU}, e_{O}=0.1$, and $\Delta r=100 \mathrm{~m}$, compared to the circular $\left(e_{O}=0\right)$ case. Figure 3 displays the minimum, average, and maximum accelerations for various eccentricities. Scaling for other parameters is simply given by the above equations. Equation (5) is maximum (in amplitude) at periapsis $\left(\theta_{O}=0\right)$, taking the value $-G M \frac{\Delta r}{p_{O}^{3}}\left(1+e_{O}\right)^{3}\left(3+e_{O}\right)$, with the semilactus rectum of the Optics spacecraft $p_{O}=a_{O}\left(1-e_{O}^{2}\right)$. Equation (5) is minimum (in amplitude) at apoapsis $\left(\theta_{O}=\pi\right)$, taking the value $-G M \frac{\Delta r}{p_{O}^{3}}\left(1-e_{O}\right)^{3}\left(3-e_{O}\right)$. The minimum/maximum of Equation (6) are the same in absolute value, and found at $\theta=\arccos \left(\frac{\sqrt{48 e^{2}+1}-1}{8 e}\right)$ and $\theta=2 \pi-\arccos \left(\frac{\sqrt{48 e^{2}+1}-1}{8 e}\right)$.

Generally, $-3 \frac{G M}{r_{O}^{3}} \Delta r$ (Equation (5) for $e_{O}=0$ ) is a good estimate for the amplitude of the tidal/orbital disruptive force for orbits with low to medium 

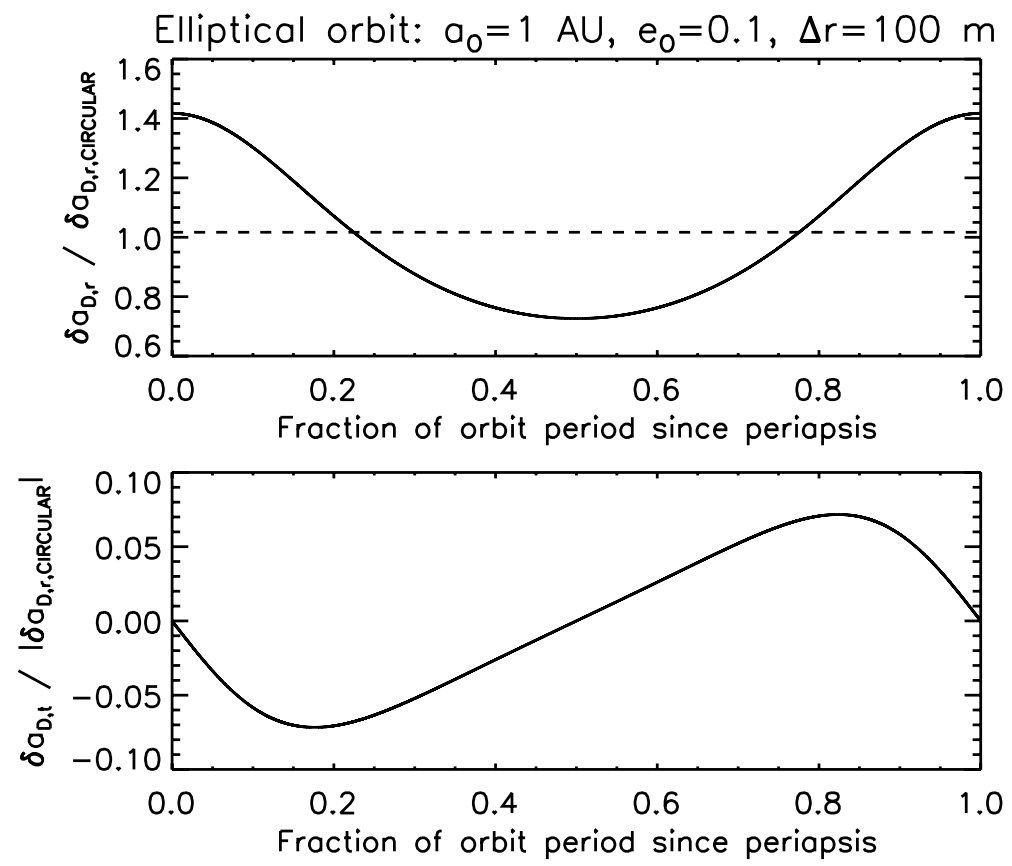

Fig. 2 Radial and tangential accelerations required by Detector spacecraft to maintain the Target-OpticsDetector alignment, due to orbital eccentricity only. The dashed line denotes the time-average of $\delta a_{D, r}$ over a full orbit

eccentricities, but also of the amplitude (though with direction varying with orbit phase) of the disruption that a telescope pointing at cosmic targets would have to overcome.

\section{Differential Acceleration due to Tidal Effects from Other Celestial Bodies}

Generally, any celestial body will exert a tidal disrupting force on the formation:

$$
\begin{aligned}
\delta a^{\text {tidal }} & =G M\left(\frac{1}{d_{O}^{2}}-\frac{1}{d_{D}^{2}}\right) \\
& \approx-2 \frac{G M}{d_{O}^{3}} \Delta d_{\text {radial }}, \Delta d_{\text {radial }} \ll d_{O} \\
& \approx-1.4 \times 10^{-9}\left(\frac{M}{M_{E a r t h}}\right)\left(\frac{385 \mathrm{Mm}}{d_{O}}\right)^{3}\left(\frac{\Delta d_{\text {radial }}}{100 \mathrm{~m}}\right) \quad\left[\mathrm{m} / \mathrm{s}^{2}\right] \\
& \approx-44\left(\frac{M}{M_{\text {Earth }}}\right)\left(\frac{385 \mathrm{Mm}}{d_{O}}\right)^{3}\left(\frac{\Delta d_{\text {radial }}}{100 \mathrm{~m}}\right) \quad[\mathrm{mm} / \mathrm{s} / \text { year }] \\
& \approx-0.25\left(\frac{M}{M_{\text {Sun }}}\right)\left(\frac{1 \mathrm{AU}}{d_{O}}\right)^{3}\left(\frac{\Delta d_{\text {radial }}}{100 \mathrm{~m}}\right) \quad[\mathrm{mm} / \mathrm{s} / \text { year }]
\end{aligned}
$$



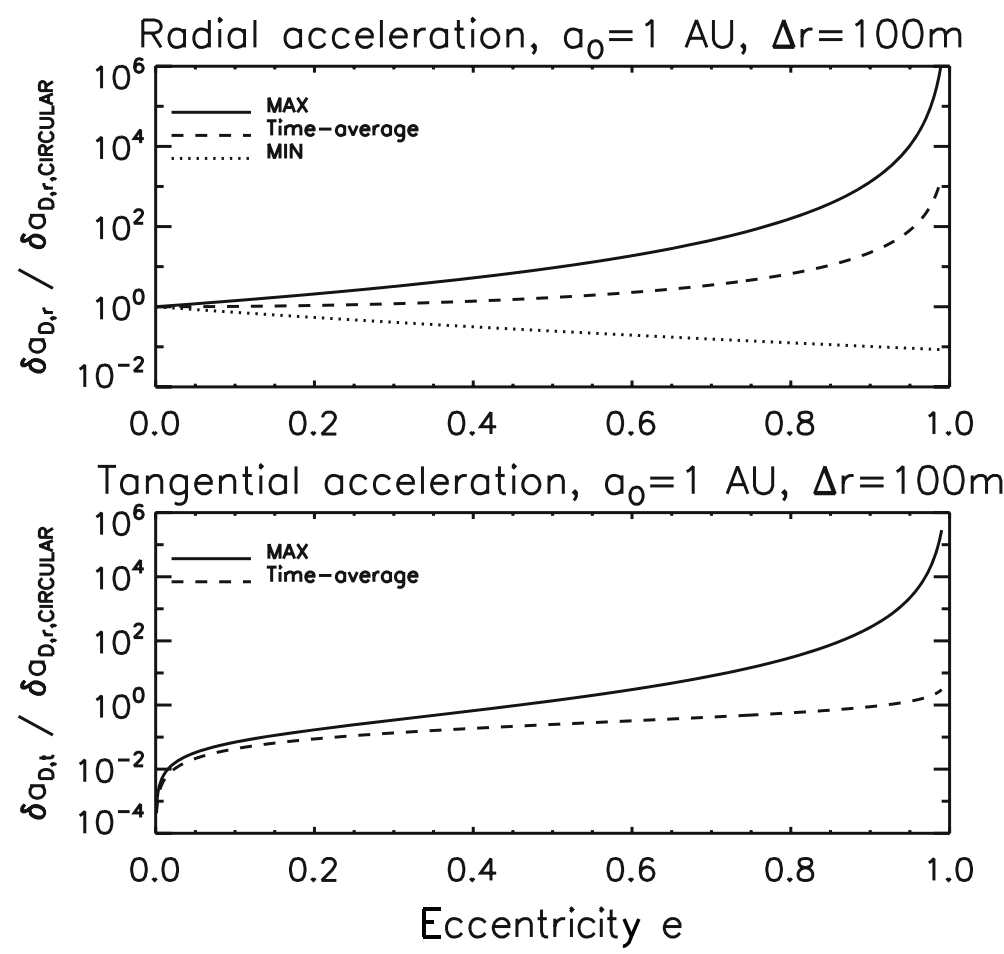

Fig. 3 Maximal, minimal, and time-averaged radial and tangential acceleration needed to compensate for elliptical orbit of telescope, relative to circular case with same semi-major axis $a_{O}$

Where $d_{O, D}$ are the distances between the disruptive body and the Optics spacecraft and Detector spacecraft, and where $\Delta d_{\text {radial }}$ is the difference in radial distance to the disrupting mass (positive corresponding to the Optics spacecraft being closest). We here deliberately used the notation " $d$ " instead of " $r$ " to emphasize that it is not the distance to the telescope orbit's center of mass. Note that the tidal disruptive effects due to the orbited target are already included in the results $\delta a_{D}^{\mathrm{NK}}$ of "Thrust the Detector Spacecraft must Exert to Sustain its non-Keplerian ("NK") Orbit".

\section{Differential Acceleration due to Solar Radiation Pressure}

The formation is influenced by the difference in solar radiation pressure experienced by the Optics spacecraft and the Detector spacecraft. In fact, far from celestial bodies, it is often the dominant effect disrupting the telescope formation.

The radiation pressure on a surface of cross-sectional area $A$ is given by:

$$
F^{\mathrm{RP}}=m \cdot a^{\mathrm{RP}}=\frac{1}{c}\left(\frac{L}{4 \pi D^{2}}\right) A \cdot(1+\alpha),
$$

where $D$ is the distance to the Sun, $L$ the solar luminosity, and $\alpha$ the albedo coefficient. For clarity, $\alpha$ (as well as the squared cosine of the incidence angle) are thereon 
assumed folded in $A$. If the target is the Sun, the difference in solar pressure between distances $r_{O}$ and $r_{D}$ is compensated by:

$$
\begin{aligned}
\delta a_{D, r}^{\mathrm{RP}} & =\frac{L}{4 \pi c}\left(\frac{A_{O}}{m_{O}} \frac{1}{r_{O}^{2}}-\frac{A_{D}}{m_{D}} \frac{1}{r_{D}^{2}}\right) \\
& =\frac{L}{4 \pi r_{O}^{2} c}\left(\frac{A_{O}}{m_{O}}-\frac{A_{D}}{m_{D}} \frac{r_{O}^{2}}{r_{D}^{2}}\right) \\
& =\frac{L}{4 \pi r_{O}^{2} c} \Delta \frac{1}{b} \\
& =4.6 \times 10^{-8}\left(\frac{1 \mathrm{AU}}{r_{O}}\right)^{2}\left(\frac{\Delta \frac{1}{b}}{1 \mathrm{dm}^{2} / \mathrm{kg}}\right) \quad\left[\mathrm{m} / \mathrm{s}^{2}\right] \\
& =1.44\left(\frac{1 \mathrm{AU}}{r_{O}}\right)^{2}\left(\frac{\Delta \frac{1}{b}}{1 \mathrm{dm}^{2} / \mathrm{kg}}\right) \quad[\mathrm{m} / \mathrm{s} / \text { year }]
\end{aligned}
$$

Where the modified inverse ballistic coefficient differential:

$$
\begin{aligned}
\Delta \frac{1}{b} & =\frac{A_{O}}{m_{O}}-\frac{A_{D}}{m_{D}}\left(\frac{r_{O}}{r_{D}}\right)^{2} \\
& \approx \frac{A_{O}}{m_{O}}-\frac{A_{D}}{m_{D}}\left(1+\frac{2 \Delta r}{r_{O}}\right) \text { when } \Delta r \ll r_{O} \\
& \approx\left(\frac{A_{O}}{m_{O}}-\frac{A_{D}}{m_{D}}\right)
\end{aligned}
$$

and will likely lie in the $1-10 \mathrm{dm}^{2} / \mathrm{kg}$ range for ordinary spacecraft pairs.

Nominally, if $\frac{A_{O}}{m_{O}}>\frac{A_{D}}{m_{D}}\left(\frac{r_{O}}{r_{D}}\right)^{2}$, then $a_{D, r}^{\mathrm{RP}}>0$, i.e. an anti-sunward thrust by the Detector spacecraft is required to maintain formation (or a sunward thrust by the Optics spacecraft). However, a judicious choice for inverse ballistic coeffient differential can, in theory, lead to $\delta a_{D, r}^{\mathrm{RP}}$ being equal in amplitude but opposite in sign to $\delta a_{D, r}^{\mathrm{NK}}$, negating the need for any radial thrust requirement by either spacecraft to maintain formation, at least to first order (minor corrections due to perturbations, sensory input errors, thruster impulse bits accuracies, etc will of course be necessary). E.g., for $\Delta r=100 \mathrm{~m}$ and $m_{D}=m_{O}=1 \mathrm{~kg}$, a $2.6 \mathrm{~mm}^{2}$ difference in cross-sectional area is enough to compensate for the Detector spacecraft's excess centrifugal acceleration and maintain the PFF. (For $\Delta r=1 \mathrm{Mm}$, that would be $2.6 \mathrm{dm}^{2}$; for $\Delta r=385 \mathrm{Mm}$ (a lunar distance), that would be $1 \mathrm{dm}^{2}$ ). Pointing errors can lead to a non-negligible sideways (tangential) thrust, which will have to be accounted for ("solar sail" effect).

Whatever avenue is chosen to compensate for the centrifugal force, it is likely that additional thrusters in the tangential and vertical directions will also be needed to keep the Target-Optics-Detector alignment and Optics-Detector distance as perfect as possible. 


\section{Differential Acceleration due to Drag in Ram Direction}

$$
\begin{aligned}
\delta a_{D, t}^{\mathrm{ram} \text { drag }} & =\frac{\rho_{O}}{2} v_{t, O}^{2}\left(\frac{A_{O}^{\mathrm{ram}}}{m_{O}}\right)-\frac{\rho_{D}}{2} v_{t, D}^{2}\left(\frac{A_{D}^{\mathrm{ram}}}{m_{D}}\right) \\
& =\frac{G M}{r_{O}}\left[\frac{\rho_{O}}{2} \frac{A_{O}^{\mathrm{ram}}}{m_{O}}-\frac{\rho_{D}}{2} \frac{A_{D}^{\mathrm{ram}}}{m_{D}} \frac{r_{D}^{2}}{r_{O}^{2}}\right] \\
& =\frac{\rho_{O}}{2} \frac{G M}{r_{O}}\left[\frac{A_{O}^{\mathrm{ram}}}{m_{O}}-\frac{A_{D}^{\mathrm{ram}}}{m_{D}}\right] \\
& \approx 7.4 \times 10^{-15}\left(\frac{1 \mathrm{AU}}{r_{O}}\right)^{3}\left(\frac{M}{M_{s u n}}\right)\left(\frac{\Delta_{b^{\mathrm{ram}}}}{1 d m^{2} / k g}\right) \quad\left[\mathrm{m} / \mathrm{s}^{2}\right] \\
& \approx 0.23\left(\frac{1 \mathrm{AU}}{r_{O}}\right)^{3}\left(\frac{M}{M_{\text {sun }}}\right)\left(\frac{\Delta_{b^{\mathrm{ram}}}}{1 d m^{2} / k g}\right) \quad[\mu \mathrm{m} / \mathrm{s} / \text { year }]
\end{aligned}
$$

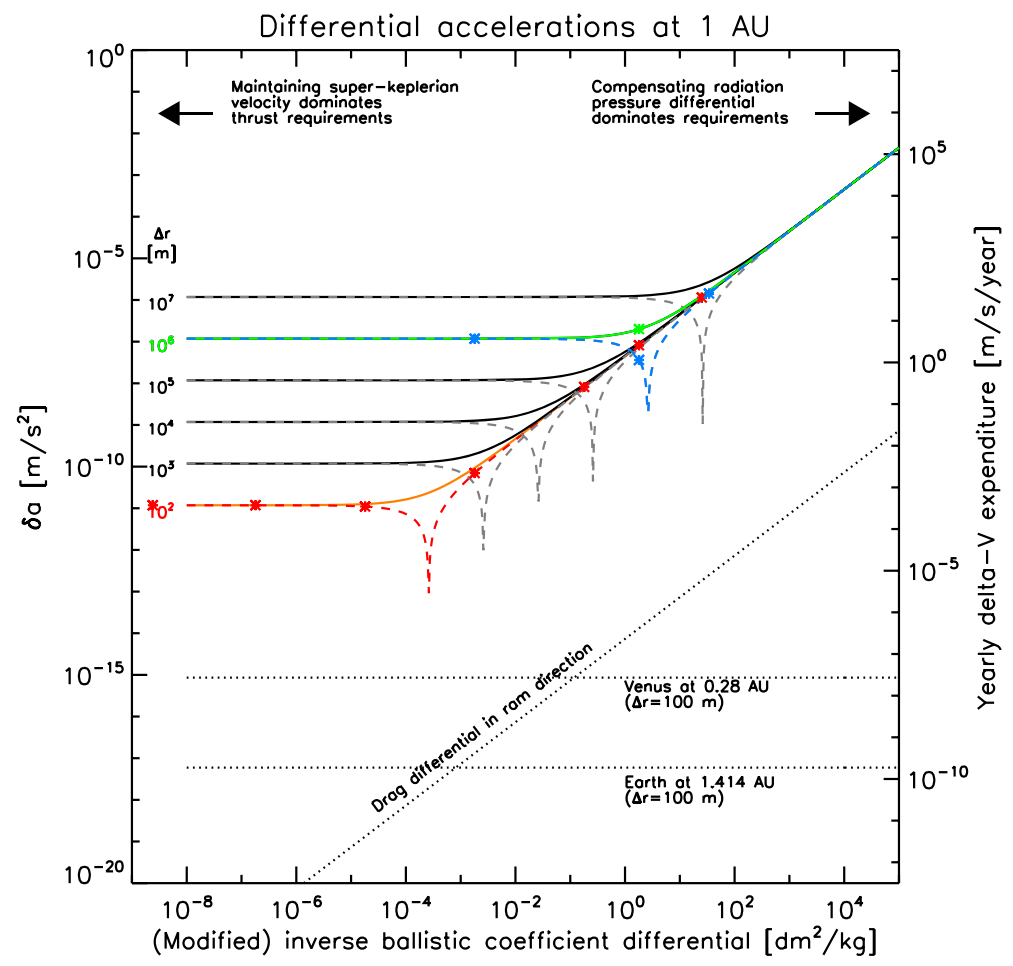

Fig. 4 Differential accelerations (and Delta-V requirement to compensate) on an Optics-Detector pair around the Sun, trying to maintain the Target-Optics-Detector alignment and Optics-Detector distance. The two spacecrafts are at $1 \mathrm{AU}, 90^{\circ}$ behind Earth. Solid lines are theoretical computations from the equations presented in the main text for $\Delta \frac{1}{b}<0$, while dashed lines correspond to $\Delta \frac{1}{b}>0$ situations. The stars are the results of simulations $3 \mathrm{a}-\mathrm{f}$ and $4 \mathrm{a}-\mathrm{c}$, all squarely falling on the theoretically-predicted curves. At low $\Delta \frac{1}{b}$, the curve branches out to various $\Delta r$ 
With, for interplanetary space and when $r_{O}$ is the distance to the sun, $\rho_{O} \approx$ $1.67 \times 10^{-24}\left(\frac{1 \mathrm{AU}}{r_{O}}\right)^{2} \mathrm{~g} / \mathrm{cm}^{3}$ and $\rho_{D}=\rho_{O}\left(\frac{r_{O}}{r_{D}}\right)^{2}$. As before, details of the the drag coefficient $C_{\mathrm{D}}$ and exact incidence angle have been folded into $A^{\text {ram }}$. This component is typically negligible far from planetary atmospheres or the Sun.

\section{Simulations}

Figure 4 summarizes differential acceleration effects on a Optics-Detector formation at $1 \mathrm{AU}$, in a heliocentric Earth-trailing orbit 90 degrees behind Earth. The crosses represent actual simulation points (note the excellent agreement with the theoretical curves), computed with the General Mission Analysis Tool (GMAT, https:// opensource.gsfc.nasa.gov/projects/GMAT/index.php) developed by NASA's Goddard Space Flight Center, which include effects from all planets and solar radiation pressure. The simulations were made assuming the Optics spacecraft orbits freely, while the Detector spacecraft is initially in the appropriate configuration (proper tangential velocity to maintain the Target-Optics-Detector alignment). All simulation results are summarized in Tables 1 and 2. Note that simulations 5a) and 5b) were made at the Earth-Sun L1 point, which requires additional station-keeping Delta-V expenditure ( $\sim 14 \mathrm{~m} / \mathrm{s} /$ year for each spacecraft) besides what is required purely to maintain the Target-Optics-Detector alignment (Fig. 5). (A set of simulations at 0.87 AU were also made, but are not shown here since they agree quantitatively with the 1 AU case discussed above.)

Table 1 Simulation configurations and total Delta-V expenditure. A 0.8 coefficient of reflectivity was used for the computations of $\Delta \frac{1}{b}$. ${ }^{*}$ : Earth-Sun L1 station-keeping requires an additional $14.2 \mathrm{~m} / \mathrm{s} /$ year for each spacecraft

\begin{tabular}{|c|c|c|c|c|c|c|c|c|}
\hline Case & $\begin{array}{l}a \\
{[\mathrm{AU}]}\end{array}$ & $e$ & $\begin{array}{l}\Delta r \\
{[\mathrm{~m}]}\end{array}$ & $\begin{array}{l}m_{o} \\
{[\mathrm{~kg}]}\end{array}$ & $\begin{array}{l}m_{d} \\
{[\mathrm{~kg}]}\end{array}$ & $\begin{array}{l}A_{o} \\
{\left[\mathrm{dm}^{2}\right]}\end{array}$ & $\begin{array}{l}A_{d} \\
{\left[\mathrm{dm}^{2}\right]}\end{array}$ & $\begin{array}{l}\text { Total } \Delta V \\
{[\mathrm{~mm} / \mathrm{s} / \mathrm{yr}]}\end{array}$ \\
\hline 3a) & 1 & 0 & 100 & 1 & 1 & 1 & 1 & 0.373 \\
\hline $3 b)$ & $"$ & $"$ & $"$ & 100 & 100 & 1.00001 & 1 & 0.373 \\
\hline $3 c)$ & $"$ & ” & $"$ & 1 & 1 & 1.00001 & 1 & 0.347 \\
\hline $3 d)$ & $"$ & $"$ & $"$ & 100 & 100 & 1.1 & 1 & 2.203 \\
\hline $3 e)$ & $"$ & ” & $"$ & 1 & 1 & 1.1 & 1 & 257.1 \\
\hline 3f) & $"$ & $"$ & $"$ & 1 & 1 & 2 & 1 & 2574.3 \\
\hline $3 \mathrm{~h})$ & $"$ & 0.01 & $"$ & 1 & 1 & 2 & 1 & 2576.91 \\
\hline $3 i)$ & $"$ & 0.1 & $"$ & 1 & 1 & 2 & 1 & 2611.65 \\
\hline 4a) & 1 & 0 & $10^{6}$ & 1 & 1 & 1.001 & 1 & 3717.98 \\
\hline $4 b)$ & $"$ & $"$ & $"$ & 100 & 100 & 200 & 100 & 1145.72 \\
\hline $\left.4 b^{\prime}\right)$ & $"$ & $"$ & $"$ & 100 & 100 & 100 & 200 & 6295.3 \\
\hline $4 c)$ & $"$ & $"$ & $"$ & 1 & 1 & 20 & 1 & 45178 \\
\hline 5a) & L1 & N/A & 100 & 1 & 1 & 1.1 & 1 & $132.16^{*}$ \\
\hline $5 b)$ & $"$ & N/A & $"$ & 1 & 1 & 1.05 & 1 & $98.29^{*}$ \\
\hline
\end{tabular}


Table 2 Simulations summary: Delta-V and deflections. To estimate constant acceleration values, divide the $\Delta V$ impulse bits presented here by the 2.4 hour time interval between them

\begin{tabular}{|c|c|c|c|c|c|c|}
\hline \multirow[t]{2}{*}{ Case } & \multicolumn{3}{|c|}{ Radial $\Delta V[\mathrm{~mm} / \mathrm{s}]$} & \multicolumn{3}{|c|}{ Transverse $\Delta V[\mathrm{~mm} / \mathrm{s}]$} \\
\hline & Max & Avg & Stddev & Max & Avg & Stddev \\
\hline 3a) & $1.08 \times 10^{-4}$ & $1.02 \times 10^{-4}$ & $4.89 \times 10^{-6}$ & $8.5 \times 10^{-6}$ & $6.65 \times 10^{-6}$ & $1.23 \times 10^{-6}$ \\
\hline 3b) & $1.08 \times 10^{-4}$ & $1.02 \times 10^{-4}$ & $4.96 \times 10^{-6}$ & $8.67 \times 10^{-6}$ & $6.62 \times 10^{-6}$ & $1.34 \times 10^{-6}$ \\
\hline 3c) & $1.01 \times 10^{-4}$ & $9.49 \times 10^{-5}$ & $4.88 \times 10^{-6}$ & $8.56 \times 10^{-6}$ & $6.70 \times 10^{-6}$ & $1.17 \times 10^{-6}$ \\
\hline $3 d)$ & $6.10 \times 10^{-4}$ & $6.03 \times 10^{-4}$ & $6.63 \times 10^{-} 6$ & $8.67 \times 10^{-6}$ & $6.78 \times 10^{-6}$ & $1.13 \times 10^{-6}$ \\
\hline 3e) & $7.05 \times 10^{-2}$ & $7.04 \times 10^{-2}$ & $3.22 \times 10^{-4}$ & $2.05 \times 10^{-5}$ & $6.61 \times 10^{-6}$ & $1.32 \times 10^{-6}$ \\
\hline 3f) & 0.705 & 0.705 & $3.22 \times 10^{-3}$ & $2.03 \times 10^{-4}$ & $6.62 \times 10^{-6}$ & $2.19 \times 10^{-6}$ \\
\hline 3h) & 0.72 & 0.706 & $1.05 \times 10^{-2}$ & $2.03 \times 10^{-4}$ & $2.29 \times 10^{-5}$ & $1.34 \times 10^{-6}$ \\
\hline 3i) & 0.876 & 0.714 & 0.101 & $6.01 \times 10^{-4}$ & $2.15 \times 10^{-4}$ & $1.31 \times 10^{-4}$ \\
\hline 4a) & 1.02 & 1.02 & $4.65 \times 10^{-3}$ & $2.90 \times 10^{-4}$ & $1.74 \times 10^{-5}$ & $1.15 \times 10^{-5}$ \\
\hline $4 b)$ & 0.314 & 0.314 & $1.43 \times 10^{-3}$ & $8.95 \times 10^{-5}$ & $2.04 \times 10^{-5}$ & $1.27 \times 10^{-5}$ \\
\hline $\left.4 b^{\prime}\right)$ & 1.725 & 1.725 & $7.88 \times 10^{-3}$ & $4.91 \times 10^{-4}$ & $1.74 \times 10^{-5}$ & $1.20 \times 10^{-5}$ \\
\hline 4c) & 12.38 & 12.38 & $5.67 \times 10^{-2}$ & $3.54 \times 10^{-3}$ & $1.19 \times 10^{-4}$ & $6.61 \times 10^{-5}$ \\
\hline 5a) & $3.86 \times 10^{-2}$ & $3.62 \times 10^{-2}$ & $1.58 \times 10^{-3}$ & $2.57 \times 10^{-4}$ & $1.02 \times 10^{-4}$ & $5.80 \times 10^{-5}$ \\
\hline $5 b)$ & $3.75 \times 10^{-2}$ & $2.69 \times 10^{-2}$ & $8.76 \times 10^{-3}$ & $2.50 \times 10^{-4}$ & $1.01 \times 10^{-4}$ & $5.52 \times 10^{-5}$ \\
\hline \multirow[t]{2}{*}{ Case } & \multicolumn{3}{|c|}{ Radial deflection [mm] } & \multicolumn{3}{|c|}{ Transverse deflection $[\mathrm{mm}]$} \\
\hline & $\operatorname{Max}$ & Avg & Stddev & $\operatorname{Max}$ & Avg & Stddev \\
\hline 3a) & 6.95 & 2.32 & 1.161 & 7.71 & 2.91 & 1.86 \\
\hline 3b) & 8.69 & 3.25 & 2.11 & 10.61 & 4.31 & 2.97 \\
\hline 3c) & 8.12 & 2.18 & 1.69 & 6.28 & 2.91 & 1.46 \\
\hline 3d) & 6.17 & 2.42 & 1.47 & 8.11 & 3.61 & 1.88 \\
\hline 3e) & 8.89 & 2.68 & 1.86 & 6.96 & 3.28 & 1.8 \\
\hline 3f) & 8.65 & 2.43 & 1.54 & 9.29 & 3.44 & 2.21 \\
\hline 3h) & 2639.2 & 961.9 & 570.1 & 8.59 & 2.24 & 1.58 \\
\hline 3i) & 18607.1 & 8976.1 & 4325.3 & 240.63 & 114.8 & 54.25 \\
\hline 4a) & 25.66 & 11.01 & 6.77 & 10.62 & 4.56 & 2.26 \\
\hline 4b) & 14.42 & 5.23 & 3.27 & 9.62 & 4.15 & 2.12 \\
\hline $4 b^{\prime}$ & 80.61 & 46.78 & 21.49 & 9.65 & 3.86 & 2.36 \\
\hline 4c) & 85.1 & 13.66 & 20.99 & 11.4 & 5.63 & 2.5 \\
\hline 5a) & 2141.1 & 1138.3 & 533.85 & 23.33 & 6.76 & 6.51 \\
\hline 5b) & 2012.7 & 716.42 & 586.03 & 17.48 & 2.59 & 2.54 \\
\hline
\end{tabular}

The details of the simulations and the control algorithm used are detailed in Appendix B. They perform a sanity check on the analytical results displayed here, and provide coarse upper limits on Detector spacecraft deflections, which are sub-cm with the control algorithm used. A full control simulation, including sensor precision and actuator accuracy are beyond the scope of the present work. 


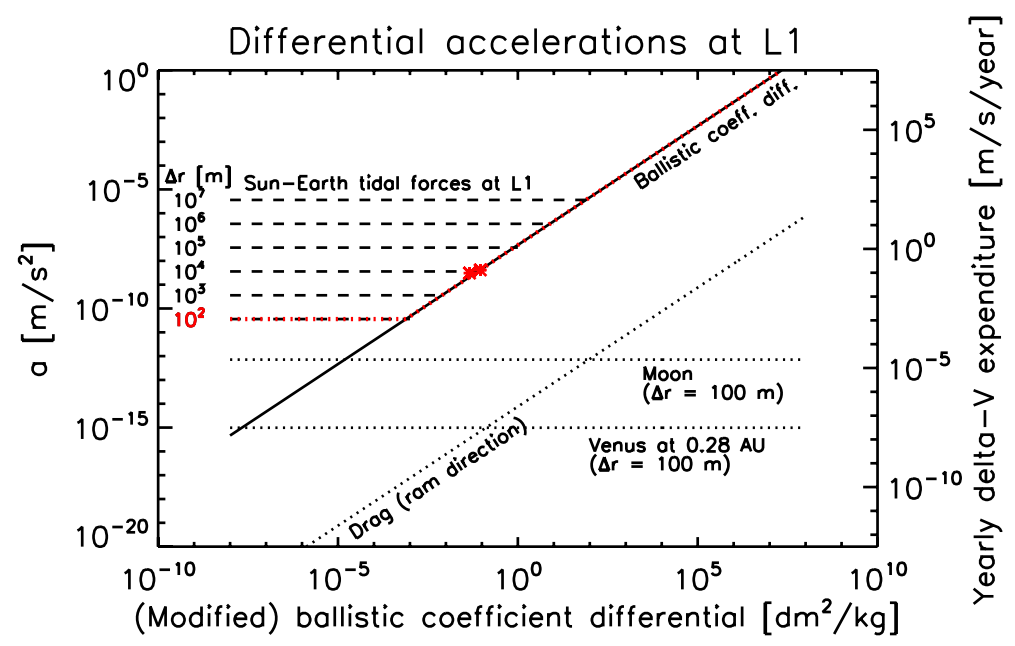

Fig. 5 Similar to Fig. 4, for a formation at the Earth-Sun L1. Note that, in addition to the formation maintenance requirement shown in this plot, each spacecraft had to expend $\sim 14 \mathrm{~m} / \mathrm{s} /$ year to be able to stay at L1

\section{Discussion}

Simple simulations have corroborated analytical predictions on Delta- $\mathrm{V}$ expenditures, and have led to deflections of up to a few millimiters in either radial or tangential directions. It is likely that, given sufficiently precise sensory input, pointing and actuator control, a rigorous control algorithm coupled with a gentle constant-thrust or solar sailing approach could achieve even smaller deflections. In near-circular scopocentric orbits, the primary thrust to maintain PFF is unidirectional (either sunward or anti-sunward), while the secondary thrusting or solar sailing in the other five directions is required to correct tiny errors, and can be several orders of magnitude less in amplitudes (e.g. Table 2).

When sufficiently far from orbital and tidal effects, differences in inverse ballistic coefficient to incident solar radiation pressure between the two spacecrafts dominate the dynamics. Only in cases with extremely large spacecraft separation $\Delta r$ (such as could be expected from e.g. a Fresnel lens-based telescopes for $\gamma$-rays) are orbital effects still the dominant disruptive forces on the formation.

Table 3 gives an overview of some currently accessible regions of space. Note that, currently or in coming years, CubeSats and SmallSats can be expected to be given regular rideshares to orbits more propitious to PFF telescopes, or to regions from which it is easier to get to such orbits: GTO, GEO, Mars Transfer Orbits, Earth-Sun L1/L2, and heliocentric.

Table 4 gives an overview of typical thruster capabilities which could be used to precisely maintain the telescope formation, or even reach the favored orbit from the launcher or rideshare release point. A wide variety of possibilities exist. CubeSat cold-gas thrusters tend to leak and to have large minimum impulse bits, making precise formation maintenance more difficult than with a less impulsive approach. Weak 
Table 3 Some rideshare-accessible orbits and time-averaged formation maintenance thrust requirements for separation $\Delta r=100 \mathrm{~m}$. Delta-V numbers in the second column are for impulsive maneuvers (low-thrust maneuvers often require in the neighborhood of twice as much)

\begin{tabular}{|c|c|c|c|}
\hline Orbit & $\begin{array}{l}\text { Typically reached } \\
\text { via... }\end{array}$ & $\begin{array}{l}\text { PFF maintenance } \\
\text { requirements }\end{array}$ & Other notes \\
\hline $\begin{array}{l}\text { LEO } \\
(600 \mathrm{~km}, \mathrm{e}=0)\end{array}$ & & $3.5 \times 10^{-4} \mathrm{~m} / \mathrm{s}^{2}$ & $\begin{array}{l}\text { Dominated } \\
\text { by orbital effects }\end{array}$ \\
\hline $\begin{array}{l}\text { LEO } \\
(\mathrm{a}=8 \mathrm{Mm}, \mathrm{e}=0.2)\end{array}$ & & $\begin{array}{l}2.3 \times 10^{-4} \mathrm{~m} / \mathrm{s}^{2} \\
(\times 2 \text { at perigee })\end{array}$ & $\begin{array}{l}\text { Dominated } \\
\text { by orbital effects }\end{array}$ \\
\hline $\begin{array}{l}\text { GTO }(a=24.6 \mathrm{Mm}, \\
\mathrm{e}=0.72)\end{array}$ & $\mathrm{LEO}+2.5 \mathrm{~km} / \mathrm{s}$ & $\begin{array}{l}3 \times 10^{-5} \mathrm{~m} / \mathrm{s}^{2} \\
(\text { perigee: } \times 14.5 \text { ) }\end{array}$ & $\begin{array}{l}\text { Dominated } \\
\text { by orbital effects }\end{array}$ \\
\hline $\begin{array}{l}\text { GEO } \\
(\mathrm{a}=42 \mathrm{Mm}, \mathrm{e}=0)\end{array}$ & $\mathrm{GTO}+1.6 \mathrm{~km} / \mathrm{s}$ & $1.6 \times 10^{-6} \mathrm{~m} / \mathrm{s}^{2}$ & $\begin{array}{l}\text { Dominated } \\
\text { by orbital effects }\end{array}$ \\
\hline $\begin{array}{l}\text { Gateway halo orbit } \\
\mathrm{a}=38.2 \mathrm{Mm}, \mathrm{e}=0.88\end{array}$ & $\mathrm{LEO}+3.6 \mathrm{~km} / \mathrm{s}$ & $\begin{array}{l}2.6 \times 10^{-7} \mathrm{~m} / \mathrm{s}^{2} \\
\text { (perigee: } \times 68 \text { ) }\end{array}$ & $\begin{array}{l}\text { Dominated } \\
\text { by orbital effects }\end{array}$ \\
\hline Earth-Sun L1/L2 & $\mathrm{LEO}+3.7 \mathrm{~km} / \mathrm{s}$ & $\begin{array}{l}\approx 10^{-6} \mathrm{~m} / \mathrm{s}^{2} \\
\text { for each } \mathrm{SC}\end{array}$ & $\begin{array}{l}\text { Dominated by } \\
\text { L1/L2 station-keeping }\end{array}$ \\
\hline $\begin{array}{l}\text { Mars Transfer Orbit } \\
(\mathrm{a}=1.26 \mathrm{AU}, \mathrm{e}=0.21)\end{array}$ & $\mathrm{LEO}+3.8 \mathrm{~km} / \mathrm{s}$ & $\begin{array}{l}3 \times 10^{-8} \mathrm{~m} / \mathrm{s}^{2} \text { for } \\
\Delta \frac{1}{b}=1 \mathrm{dm}^{2} / \mathrm{kg}\end{array}$ & $\begin{array}{l}\text { Dominated by } \\
\text { radiation pressure }\end{array}$ \\
\hline $\begin{array}{l}\text { Heliocentric orbit } \\
(\mathrm{a}=1 \mathrm{AU}, \mathrm{e} \approx 0)\end{array}$ & $\begin{array}{l}\text { LEO+3.2 km/s, } \\
\text { GTO+0.7 km/s } \\
(+0.5-1.5 \mathrm{~km} / \mathrm{s} \\
\text { to nullify drift) }\end{array}$ & $\begin{array}{l}5 \times 10^{-8} \mathrm{~m} / \mathrm{s}^{2} \text { for } \\
\Delta \frac{1}{b}=1 \mathrm{dm}^{2} / \mathrm{kg} \\
\text { (rad. pressure) }\end{array}$ & $\begin{array}{l}\text { Earth-trailing/leading } \\
\text { or Artemis disposal } \\
\text { (lunar gravity } \\
\text { assist possible) }\end{array}$ \\
\hline $\begin{array}{l}\text { DRO } \\
(\mathrm{a}=1 \mathrm{AU}, \mathrm{e}=0.05)\end{array}$ & $\begin{array}{l}\text { Earth-Sun L1/2 } \\
+0.7-1 \mathrm{~km} / \mathrm{s}\end{array}$ & $\begin{array}{l}5 \times 10^{-8} \mathrm{~m} / \mathrm{s}^{2} \text { for } \\
\Delta \frac{1}{b}=1 \mathrm{dm}^{2} / \mathrm{kg}\end{array}$ & $\begin{array}{l}\text { Stays within } \\
\sim 0.05 \text { AU of Earth }\end{array}$ \\
\hline
\end{tabular}

but constant thrust propulsion will tend to have formation-keeping control mostly limited by other factors, such as attitude control \& knowledge, accuracy of actuators, or minimum impulse bits. Note that S/C attitude control may not need to be extraordinary for the observations themselves, as most optical systems tend to follow the "thin lens" approximation to first order, and detector plates may also be somewhat tilted with little loss.

There are of course a host of other considerations, such as the bright Sun possibly complicating tracking of the Optics spacecraft by the Detector spacecraft (though a combination of an optical camera and radio direction-finding and ranging covers quite a bit of ground), or the shadowing of the Sun by the Optics spacecraft impacting the Detector spacecraft's solar panels.

Within cislunar space, it appears that a thruster-based approach is necessary. Most tantalizing for remote-sensing solar physics and astrophysics is the possibility to have a Sun-orbiting telescope in trans-lunar orbits exclusively using solar sailing to main- 
Table 4 Some example thrusters and spacecraft masses. All numbers approximate

\begin{tabular}{|c|c|c|c|c|}
\hline Thruster & $\begin{array}{l}\text { Spacecraft } \\
\text { total mass }\end{array}$ & $\begin{array}{l}\text { Thrust-to-mass } \\
\text { ratio }\end{array}$ & $\begin{array}{l}\text { Lifetime at } \\
\text { max thrust }\end{array}$ & Notes \\
\hline $\begin{array}{l}3.6 \mathrm{~mW} \text { laser pointer } \\
\text { ("Photon drive") }\end{array}$ & $1 \mathrm{~kg}$ & $\begin{array}{l}10^{-11} \mathrm{~m} / \mathrm{s}^{2} \\
3 \times 10^{-4} \mathrm{~m} / \mathrm{s} / \mathrm{yr}\end{array}$ & $\infty$ & \\
\hline $\begin{array}{l}1 \mathrm{dm}^{2} \text { solar panel } \\
\text { at } 1 \mathrm{AU}(\alpha=0.2)\end{array}$ & $1 \mathrm{~kg}$ & $\begin{array}{l}6 \times 10^{-8} \mathrm{~m} / \mathrm{s}^{2} \\
2 \mathrm{~m} / \mathrm{s} / \mathrm{yr}\end{array}$ & $\infty$ & \\
\hline $\begin{array}{l}1 \mathrm{dm}^{2} \text { reflective panel } \\
\text { at } 1 \mathrm{AU}(\alpha=1)\end{array}$ & $1 \mathrm{~kg}$ & $\begin{array}{l}10^{-7} \mathrm{~m} / \mathrm{s}^{2} \\
3 \mathrm{~m} / \mathrm{s} / \mathrm{yr}\end{array}$ & $\infty$ & \\
\hline $\begin{array}{l}\text { Commercial } \\
\text { Busek Bmp-220 } \\
\text { for CubeSats }\end{array}$ & $4 \mathrm{~kg}$ & $\begin{array}{l}3 \times 10^{-5} \mathrm{~m} / \mathrm{s}^{2} \\
1100 \mathrm{~m} / \mathrm{s} / \mathrm{yr}\end{array}$ & $\begin{array}{l}\sim 17 \\
\text { days }\end{array}$ & $\begin{array}{l}\text { Min. impulse } \\
\text { bit: } 5 \mu \mathrm{m} / \mathrm{s}\end{array}$ \\
\hline $\begin{array}{l}\text { Commercial } \\
\text { IFM Nano Thruster }\end{array}$ & $20 \mathrm{~kg}$ & $\begin{array}{l}5 \times 10^{-7} \text { to } \\
2.5 \times 10^{-5} \mathrm{~m} / \mathrm{s}^{2}\end{array}$ & $\begin{array}{l}>100 \\
\text { days }\end{array}$ & \\
\hline $\begin{array}{l}\text { Ion drive: SMART-1 } \\
\text { ( } 367 \mathrm{~kg} \text { wet; } 287 \mathrm{~kg} \text { dry) }\end{array}$ & $300 \mathrm{~kg}$ & $\begin{array}{l}2 \times 10^{-4} \mathrm{~m} / \mathrm{s}^{2} \\
5400 \mathrm{~m} / \mathrm{s} / \mathrm{yr}\end{array}$ & $0.5 \mathrm{yr}$ & \\
\hline
\end{tabular}

tain the PFF (Tables 3 \& 4). A combination of flaps, rudders, or even solar panels with adjutable orientation and inclinations could maintain the PFF. Such a telescope would have no consumables. Drifting Earth-trailing or Earth-leading orbits are easier to reach than Earth-Sun L1/L2, and have been used by the Spitzer, Kepler, and STEREO missions (through Delta-II rocket launches). Such orbits should also be available to SmallSats in the coming years thanks in part to rideshares on the upcoming SLS (Artemis) launches to the Moon ("heliocentric disposal orbits"). Research into nullifying the drift, i.e. preserving the Earth-telescope distance (and downlink rate) over years, is presented in e.g. [7] and require in the neighborhood of $\sim 1 \mathrm{~km} / \mathrm{s}$ Delta-V. Distant Retrograde Orbits (DRO) appear to be a robust alternative. They are accessible at a somewhat greater Delta-V cost. DROs have basically the same semi-major axis (and orbital period) as Earth and the same heliocentric orbital phase (anomaly), but with a slightly higher eccentricity, giving the impression the spacecrafts are orbiting the Earth-Moon system in a retrograde fashion. Stable DROs (requiring no station keeping) starting at a few percent of an AU away the from Earth-Moon system exist, but likely require a $0.5-1 \mathrm{~km} / \mathrm{s}$ Delta-V to reach from Earth-Sun L1 or L2.

\section{Conclusions}

Through linearized relative equations of motion, we have gauged the feasibility of a Precise Formation Flying telescope, and have been particularly interested in the Sun as a target. We also present exact analytical equations in Appendix A. 
Simulations spanning a wide range of parameters have fully supported both the cases of heliocentric and Earth-Sun L1 orbits. In most cases presented here, the Detector spacecraft drifts are sufficiently small that detector area does not need to be much larger than conventional, if at all (Table 2).

Translunar space is an ideal region to use PFF telescopes. They are already accessible to large payloads on heavy launchers, and becoming available to CubeSats and SmallSats via rideshares. We suggest putting such smaller payloads in heliocentric orbits, via Artemis and other rideshares to high-energy orbits, likely in the form of a single spacecraft that eventually separates into Optics spacecraft and Detector spacecraft. It is likely that in most cases, the payload will require a $\sim 1 \mathrm{~km} / \mathrm{s}$ stage ot capability to complete the trip (e.g. L1 to DRO, or to nullify the drift away from Earth), but once in position, low-thrust propulsion and/or solar sailing can maintain the formation, potentially for years, and with $100 \%$ observation time throughout the orbit.

We shall conclude by stating that, while this study was oriented towards the Sun as target, it is relevant to any solar system target, such as planets. Cosmic targets should also be accessible for Astrophysics, with a slight increase in difficulty due to primary thrusting varying in both amplitude and direction with orbit phase.

Acknowledgments We thank Dan Cosgrove for various conversations over the years, including introducing us to DROs, and we thank both anonymous referees whose suggestions have greatly improved this work.

\section{Compliance with Ethical Standards}

Conflict of interests On behalf of all authors, the corresponding author states that there is no conflict of interest.

Open Access This article is licensed under a Creative Commons Attribution 4.0 International License, which permits use, sharing, adaptation, distribution and reproduction in any medium or format, as long as you give appropriate credit to the original author(s) and the source, provide a link to the Creative Commons licence, and indicate if changes were made. The images or other third party material in this article are included in the article's Creative Commons licence, unless indicated otherwise in a credit line to the material. If material is not included in the article's Creative Commons licence and your intended use is not permitted by statutory regulation or exceeds the permitted use, you will need to obtain permission directly from the copyright holder. To view a copy of this licence, visit http://creativecommonshorg/licenses/by/4.0/.

\section{Appendix A: Exact 2D Derivation of the Differential Thrusts Required to Maintain the PFF}

To maintain the Target-Optics-Detector alignment, both Optics spacecraft and Detector spacecraft will have to be in the same orbital plane around the target. We are hence 
here only considering moition in 2-D. A full control simulation will of course have to include out-of-plane motions.

\section{Circular Scopocentric Case}

Assuming the Optics spacecraft is freely coasting in a circular keplerian orbit around its target, its velocity (all tangential) is given by the centrifugal force and gravity canceling each other:

$$
\frac{v_{O}^{2}}{r_{O}}-\frac{G M}{r_{O}^{2}}=0
$$

Leading to:

$$
v_{\mathrm{O}}=\sqrt{\frac{G M}{r_{O}}}
$$

where $M$ the mass of the orbited target (e.g., the Sun), $G$ is Newton's gravitational constant, $r_{O}$ is the Optics spacecraft-target distance (e.g. $1 \mathrm{AU}$ ). To preserve the Target-Optics-Detector alignment, the angular velocities around the Sun of both Optics spacecraft and Detector spacecraft must be the same, i.e. $\omega_{O}=\omega_{D}$, leading to:

$$
\frac{v_{O}}{r_{O}}=\frac{v_{D}}{r_{D}}=\frac{v_{D}}{r_{O}+\Delta r}
$$

where $\Delta r=r_{D}-r_{O}$.

Let's assume that $v_{D}$ is initially set to that value. If allowed to coast, the Detector spacecraft would enter an elliptical orbit, eventually destroying the TargetOptics-Detector alignment. In terms of forces (or accelerations), there is an excess centrifugal force for the Detector spacecraft, and the Detector spacecraft thrust required to compensate it is given by:

$$
\begin{aligned}
& \delta a_{D, r}^{\mathrm{NK}}=\frac{G M}{r_{D}^{2}}-\frac{v_{D}^{2}}{r_{D}}<0 \\
& \delta a_{D, t}^{\mathrm{NK}}=0
\end{aligned}
$$

With zero net radial acceleration, the Optics-Detector distance is maintained, and the trajectory of the Detector spacecraft will remain circular, with an angular velocity (or orbital period) equal to the Optics spacecraft's, thus preserving both components of the PFF (Target-Optics-Detector alignment and the Optics-Detector distance) throughout the formation's orbit. 
Using $\omega_{D}=\omega_{O}$, Eqs (28) and (29) lead to:

$$
\begin{aligned}
\delta a_{D, r}^{\mathrm{NK}} & =\frac{G M}{r_{D}^{2}}-\frac{v_{D}^{2}}{r_{D}} \\
& =\frac{G M}{r_{D}^{2}}-\omega_{O}^{2} \cdot r_{D} \\
& =-\frac{G M}{\left(r_{O}+\Delta r\right)^{2}}-\frac{G M}{r_{O}^{3}} \cdot\left(r_{O}+\Delta r\right) \\
& =-G M \frac{3\left(\Delta r / r_{O}\right)+3\left(\Delta r / r_{O}\right)^{2}+\left(\Delta r / r_{O}\right)^{3}}{r_{O}^{2}\left(1+\Delta r_{O}\right)^{2}} \\
& \approx-3 G M \frac{\Delta r}{r_{O}^{3}}, \text { when } \Delta r \ll r_{O} \\
& \approx-1.2 \times 10^{-11}\left(\frac{M}{M_{\text {sun }}}\right)\left(\frac{1 \mathrm{AU}}{r_{O}}\right)^{3}\left(\frac{\Delta r}{100 \mathrm{~m}}\right)\left[\mathrm{m} / \mathrm{s}^{2}\right] \\
& \approx-0.38\left(\frac{M}{M_{\text {sun }}}\right)\left(\frac{1 \mathrm{AU}}{r_{O}}\right)^{3}\left(\frac{\Delta r}{100 \mathrm{~m}}\right)[\mathrm{mm} / \mathrm{s} / \text { year }]
\end{aligned}
$$

For $m_{D}=1 \mathrm{~kg}, M=M_{\text {sun }}, r_{O}=1 \mathrm{AU}$ and $\Delta r=100 \mathrm{~m}$, this thrust level $(12 \mathrm{pN})$ is comparable to that of a mere $3.6 \mathrm{~mW}$ laser pointer pointed anti-sunward.

Generally, Equation (35) is a good estimate for the amplitude of the tidal/orbital disrupting force for orbits with low to medium eccentricities, but also of the amplitude (though with direction varying with orbit phase) of the disruption that a telescope pointing at cosmic targets would have to overcome.

\section{General (Elliptical) Scopocentric Case}

In a co-rotating coordinate system $(r, \theta)$, a body's or spacecraft's radial and tangential accelerations are exactly given by:

$$
\begin{aligned}
\ddot{r} & =a_{r}+r \dot{\theta}^{2} \\
r \ddot{\theta} & =a_{t}-2 \dot{r} \dot{\theta}
\end{aligned}
$$

Where $a_{r}$ and $a_{t}$ are the applied radial and tangential accelerations (gravity, thrusters, solar radiation pressure,...). The Optics spacecraft is free-flying: $a_{O, r}=$ $-\frac{G M}{r_{O}^{2}}$ and $a_{t, O}=0$. The Detector spacecraft has $a_{D, r}=-\frac{G M}{r_{D}^{2}}+\delta a_{D, r}$ and $a_{D, t}=\delta a_{D, t}$, where $\delta a_{D, r}$ and $\delta a_{D, t}$ are the accelerations the Detector spacecraft requires to maintain the Target-Optics-Detector alignment and Optics-Detector distance. Using $\ddot{\theta}_{D}-\ddot{\theta}_{O}=\dot{\theta}_{D}-\dot{\theta}_{O}=\theta_{D}-\theta_{O} \equiv 0$ and $\ddot{r}_{D}-\ddot{r}_{O}=\dot{r}_{D}-\dot{r}_{O} \equiv 0$ :

$$
\delta a_{D, r}^{\mathrm{NK}}=G M\left(\frac{1}{r_{D}^{2}}-\frac{1}{r_{O}^{2}}\right)-\Delta r \dot{\theta}_{O}^{2}
$$




$$
\begin{aligned}
& =G M\left[\frac{1}{r_{D}^{2}}-\frac{1}{r_{O}^{2}}-\Delta r \frac{(1+e \cdot \cos \theta)^{2}}{r_{O}^{2} \cdot a_{O}\left(1-e^{2}\right)}\right] \\
& =G M\left[\frac{1}{r_{O}^{2}}\left(\frac{1}{1+\frac{\Delta r}{r_{O}}}-1\right)-\Delta r \frac{(1+e \cdot \cos \theta)^{2}}{r_{O}^{2} \cdot a_{O}\left(1-e^{2}\right)}\right] \\
& \approx G M\left[\frac{1}{r_{O}^{2}}\left(1-\frac{\Delta r}{r_{O}}-1\right)-\Delta r \frac{(1+e \cdot \cos \theta)^{2}}{r_{O}^{2} \cdot a_{O}\left(1-e^{2}\right)}\right] \text {, when } \Delta r \ll r_{O} \\
& \approx-G M \frac{\Delta r}{r_{O}}\left(\frac{2}{r_{O}^{2}}+\frac{(1+e \cdot \cos \theta)^{2}}{r_{O} \cdot a_{O}\left(1-e_{O}^{2}\right)}\right) \\
& \approx-G M \frac{\Delta r}{a_{O}^{3}}\left(\frac{1+e_{O} \cdot \cos \theta}{1-e_{O}^{2}}\right)^{3}\left(3+e_{O} \cdot \cos \theta\right) \\
& \left(\approx-3 G M \frac{\Delta r}{r_{O}^{3}}, \text { when furthermore } e_{O} \approx 0\right) \\
& \delta a_{D, t}^{\mathrm{NK}}=2 r_{D}\left[\frac{\dot{r}_{D}}{r_{D}} \dot{\theta}_{D}-\frac{\dot{r}_{O}}{r_{O}} \dot{\theta}_{O}\right] \\
& =2 r_{D} \dot{r}_{O} \dot{\theta}_{O}\left(\frac{1}{r_{D}}-\frac{1}{r_{O}}\right) \\
& =-2 \frac{\Delta r}{r_{O}} \dot{r}_{O} \dot{\theta}_{O} \\
& =-2 G M \frac{\Delta r}{r_{O}^{3}} \cdot e_{O} \cdot \sin \theta \\
& =-2 G M \frac{\Delta r}{a_{O}^{3}} \cdot e_{O} \cdot \sin \theta\left(\frac{1+e_{O} \cdot \cos \theta}{1-e_{O}^{2}}\right)^{3} \\
& \left(\approx 0 \text {, when } e_{O} \approx 0\right)
\end{aligned}
$$

Where we have used the well-known Keplerian relations $\dot{r}_{O}=\sqrt{\frac{G M}{p_{O}}} e \sin \theta_{O}$ and $r_{O} \dot{\theta}_{O}=\sqrt{\frac{G M}{p_{O}}}\left(1+e \cos \theta_{O}\right)$, with the semilactus rectum $p_{O}=a_{O}\left(1-e_{O}^{2}\right)$.

\section{Appendix B: Simulations Details}

Astrodynamical simulations of the optics and detector spacecraft were performed using the General Mission Analysis Tool (GMAT, https://opensource.gsfc.nasa.gov/ projects/GMAT/index.php) developed by NASA's Goddard Space Flight Center.

Two orbit shapes were considered in our investigation: a direct heliocentric orbit and an orbit of the L1 Lagrange point between the Earth and Sun (Earth-Sun L1). 
The force model employed included the Sun and all eight planets, plus the Moon and Pluto. The Earth was the only body not modeled as a point mass, using the default GMAT fourth-degree model. The solar radiation pressure model was a default spherical model included with GMAT.

In configuring the framework for the simulations, we defined a coordinate system in the Sun-Optics spacecraft rotating frame. The origin of this frame centered on the Optics spacecraft. The z-axis was defined to lie along the line connecting the Optics spacecraft and the Sun. The y-axis was situated normal to the solar ecliptic. Within this framework, the Detector spacecraft was maneuvered to maintain a specified distance along the z-axis from the Optics module, with minimal excursions in the $\mathrm{x}-\mathrm{y}$ plane, thus keeping alignment with the Optics module and the Sun. Note that if no force is applied to the Detector module, it will drift out of alignment along all three axes. To counter this drift, forces were applied in the appropriate directions so as to maintain alignment with specified tolerances.

For these thrust forces, we approximated a continuous thrust model by applying impulsive burns at 2.4 hours intervals (10 times per day), and hence close to a constant-thrust ideal case.

The key to maintaining alignment was to target each velocity $\left(v_{i-b i a s}\right)$ in each axis direction so as to match the apparent drift velocities observed in the no-thrust case ("apparent" in the Sun-Optics spacecraft frame.) This was accomplished through the GMAT "Target-Achieve" construct where each $v_{i-b i a s}$ was the target in each Achieve goal.

As the Detector spacecraft drifted away from alignment tolerance, $v_{i-b i a s}$ was adjusted by a small amount $\left(d v_{i-b i a s}\right)$ so as to regain alignment tolerance. Thus, a significant portion of the time spent configuring the simulations was consumed by the determination of the optimal initial values for each $v_{i-b i a s}$ and $d v_{i-b i a s}$ for each parameter set.

An artifact of the step-wise (versus continuous) model is the introduction of oscillations which, in order to minimize, required some small additional amount of Delta-V. Thus the Delta-V values presented herein represent an upper-limit on the actual values which would be consumed by a more sophisticated and robust continuous firing scheme. Despite this, Fig. 4 clearly shows the excellent fit between theory and simulations. It should also be noted that the control algorithm was designed with quasi-circular orbits in mind. It does very well with such, leading to deflections $<1 \mathrm{~cm}$ in both radial and tangential directions. For elliptical orbits, (simulation $3 \mathrm{i}, e=0.1$ ), the deflection becomes larger. It is very likely that a more general control algorithm would just as easily handle elliptical cases, since exact analytical formulation (or extremely accurate linearized formulations) exist (Section 1).

In the case of the Earth-Sun libration point orbit, an additional control loop was required for station-keeping purposes. The GMAT program provides an optimizer add-on which was applied to the Optics spacecraft thrusting scheme. We used the station-keeping strategy described in [8], which was to maintain zero velocity in the z-direction when crossing the z-axis (Earth-Sun Line) at the closest distance to Earth (each complete rotation). Velocity in the solar pole direction (out-of-plane) was also kept very low. Thus the overall Delta-V budget was increased by this additional (and larger) Delta-V. 
The specifics of the control program are best described by the following GMATstyle pseudo-code snippet (for our Case 3a):

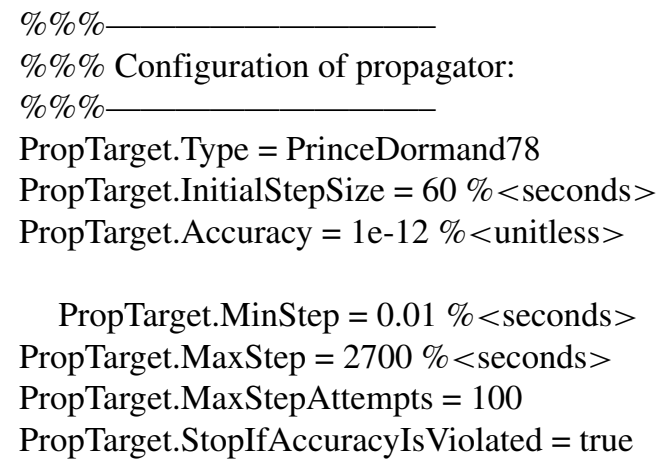

Positional_Tolerance_i $=2 \mathrm{e}-7 \%<\mathrm{km}>$

$\% \% \%$

$\% \% \%$ For each cardinal direction, $<\mathrm{i}>$, in the rotating frame:

$\% \% \%$ Set the value of applied increment to drift counter-velocities.

$\% \%$ (These values were determined by observational trial and $\% \% \%$ error.)

$\% \% \%$

$D v_{\text {i-bias }}=5 \mathrm{e}-12 \%<\mathrm{km} / \mathrm{s}>$

\footnotetext{
$<$ Begin Main Loop - Until ElapsedDays == 1200 days $>$ $\% \% \%$

$\% \% \%$ Impulsive Maneuver Targeting Block

$\% \% \%$ For each cardinal direction, $<\mathrm{i}>$, in the rotating frame:

$\% \% \%$ Thrust element is 'Varied' until the Velocity goal is reached

$\% \% \%$ within the designated Tolerance.
} 
$\% \% \%$

Vary Detector_Thrust_Element_i $=-0.001,\{$ Perturbation $=1 \mathrm{e}-5$, Lower $=-1 \mathrm{e}-2$, Upper $=1 \mathrm{e}-2$, MaxStep $=1\}$

Achieve Velocity_i $=v_{i-\text { bias }},\{$ Tolerance $=1 \mathrm{e}-12\}$

$<$ For each cardinal direction: Record Delta-V determined from Targeting Block $>$

$\% \% \%$

$\% \% \%$ Propagate for one propagation_step $=0.1$ day

$\% \%$

Propagate 'Prop' Synchronized PropTarget(Observer, Detector) ElapsedDays = propagation_step, StopTolerance $=1 \mathrm{e}-005$

$\% \% \%$

$\% \% \%$ For each cardinal direction, $<\mathrm{i}>$, in rotating frame:

$\% \% \%$ Check for violations of drift tolerance

$\% \% \%$

If abs(Position_i) $>$ Positional_Tolerance_i

$\% \% \%$

$\% \% \%$ Drift deflection tolerance is exceeded, so alter velocity_bias

$\% \% \%$ by a small amount in opposite direction of deflection

$\% \% \%$

If Position_i $>0$

$v_{i-b i a s}=v_{i-b i a s}-D v_{i-b i a s}$

If Position $\_\mathrm{i}<0$

$v_{i-b i a s}=v_{i-b i a s}+D v_{i-b i a s}$

$<$ End Main Loop $>$

$<$ End of Snippet $>$

\section{References}

1. Leyre, X., Sghedoni, M., Vives, S., Lamy, P., Pailharey, E.: Society of Photo-Optical Instrumentation Engineers (SPIE) Conference Series. In: Proceedings of SPIE Society of Photo-Optical Instrumentation Engineers (SPIE) Conference Series, vol. 5899, ed. by H.A. MacEwen, vol. 5899, pp. 221-229 (2005). https://doi.org/10.1117/12.617187

2. Vivès, S., Lamy, P., Levacher, P., Boit, J.L., Saisse, M.: Society of Photo-Optical Instrumentation Engineers (SPIE) Conference Series. In: Proceedings of SPIE Society of Photo-Optical Instrumentation Engineers (SPIE) Conference Series, vol. 6265, vol. 6265, p. 626524 (2006). https://doi.org/10. $1117 / 12.671621$

3. Dennis, B.R., Skinner, G.K., Li, M.J., Shih, A.Y.: Solar Phys. 279(2), 573 (2012). https://doi.org/10. 1007/s11207-012-0016-7

4. Clohessy, W., Wiltshire, R.: Journal of the Astronautical Sciences 27, 653 (1960)

5. Melton, R.G.: Journal of Control, Guidance, and Dynamics 23, 604 (2000) 
6. Alfriend, K.T., Vadali, S.R., Gurfil, P., How, J.P., Breger, L.S.: Spacecraft Formation Flying: Dynamics control and navigation (2010)

7. Joffre, E., Moro, V., Renk, F., Waldemar, M.: https://indico.esa.int/event/224/papers/3889/files/239ICATT2 018_-_E_Joffre_-_Astrodynamics_techniques_for_missions_towards_Earth -Trailing_or_EarthLeading_Heliocentric_Orbits.pdf (2018)

8. Folta, D.C., Woodward, M.A., Cosgrove, D., vol. 11. https://ntrs.nasa.gov/archive/nasa/casi.ntrs.nasa. gov/20110015252.pdf (2011)

Publisher's Note Springer Nature remains neutral with regard to jurisdictional claims in published maps and institutional affiliations. 